\title{
Efetividade e segurança da corticoterapia inalatória em crianças e adolescentes
}

\section{asmáticos}

\author{
Effectiveness and safety of inhaled corticosteroid therapy in asthmatic children and adolescents \\ Eficacia y seguridad del tratamiento con corticosteroides inhalados en niños y adolescentes \\ asmáticos
}

Recebido: 07/06/2021 | Revisado: 16/06/2021 | Aceito: 28/06/2021 | Publicado: 11/07/2021

\author{
Lucas Nóbrega de Oliveira \\ ORCID: https://orcid.org/0000-0003-2656-6910 \\ Residência Multiprofissional em Saúde da Criança, Brasil \\ E-mail: lucasnobrega18@outlook.com \\ Aline Kely Felício de Sousa Santos \\ ORCID: https://orcid.org/0000-0002-2720-3129 \\ Residência Multiprofissional em Saúde da Criança, Brasil \\ E-mail: alinekely.f@gmail.com \\ Richard Morrinson Couras de Carvalho \\ ORCID: https://orcid.org/0000-0002-2564-3657 \\ Residência Multiprofissional em Saúde da Criança, Brasil \\ E-mail: rich_couras@hotmail.com \\ Bruno Wesley Ramalho Cirilo Ferreira \\ ORCID: https://orcid.org/0000-0003-3448-0957 \\ Universidade Federal da Paraíba, Brasil \\ E-mail: brunnoramallho@ hotmail.com \\ Cibério Landim Macedo \\ ORCID: https://orcid.org/0000-0002-0824-4056 \\ Residência Multiprofissional em Saúde da Criança, Brasil \\ E-mail:ciberiolandim@hotmail.com
}

\begin{abstract}
Resumo
O objetivo deste estudo foi investigar o uso de corticosteroides inalatórios (CI) no tratamento da asma, em crianças e adolescentes, com a finalidade de fornecer informações relevantes e atuais para assegurar um tratamento mais seguro e efetivo. Foi realizada uma revisão sistemática na literatura disponível nas bases de dados PubMed, Scielo e ScienceDirect, utilizando como descritores: asthma, corticosteroids, drug therapy e children. Foram incluídos artigos na categoria original, disponibilizados na íntegra, publicados nos idiomas português, inglês e espanhol, com intervalo de 2016 a 2021. Dos 233 estudos encontrados, 14 foram selecionados. Na pesquisa observou-se a influência que o tamanho de partícula tem sobre a efetividade e segurança do tratamento, alterando a disponibilidade do medicamento no órgão alvo e a dosagem administrada para promover o efeito desejado. Ademais, as particularidades dos CI disponíveis no mercado que podem ser utilizados isoladamente ou em combinações com $\beta_{2}$-agonistas adrenérgicos, nas quais essas permitem uma redução da dose de CI usado, diminuindo assim a incidência de possíveis reações adversas à medicamentos (RAM). É importante ressaltar que a fluticasona mostrou-se como um dos medicamentos mais seguros quando foi analisada sua influência sobre o crescimento de crianças e adolescentes. Por último, apesar da gama de estudos na área, é necessário que novas pesquisas sobre esse tema sejam realizadas, principalmente aquelas que envolvam a influência do tamanho de partícula na efetividade e segurança do tratamento, bem como as principais RAM acerca do tema proposto.
\end{abstract}

Palavras-chave: Asma; Corticosteroides; Farmacoterapia.

\begin{abstract}
The aim of this study was to investigate the use of inhaled corticosteroids (IC) for the treatment of asthma in order to provide relevant information and ensure a safer and more effective treatment for children and teenagers. An systematic review of the literature available on PubMed, Scielo and ScienceDirect was carried out using the following the descriptors: asthma, corticosteroids, drug therapy and children. The selected works were: open access; published in either Portuguese, English or Spanish; ranging from the year 2016 to 2021. Of the 233 compiled studies, 14 were selected. In the research, it was noticed that the particle size has an influence in the effectiveness and safety of the treatment, changing the availability of the drug in its target and the dosage administered to promote the desired effect. Furthermore, the particularities of ICs available on the market, which can be used alone or in combination with $\beta 2$ adrenergic agonists, allow for a reduction in the IC dosage, thus reducing the incidence of possible adverse drug reactions (ADRs). It is important to emphasize that fluticasone proved to be one of the safest medications when its
\end{abstract}


influence in the growth of children and adolescents was considered. Finally, despite the range of studies in the area, further research on this topic is necessary, especially those involving the influence of particle size on the effectiveness and safety of treatment, as well as the main ADRs on the proposed topic.

Keywords: Asthma; Corticosteroids; Pharmacotherapy.

\section{Resumen}

El objetivo de este estudio fue investigar el uso de corticosteroides inhalados (CI) en el tratamiento del asma en niños y adolescentes, con el fin de proveer información relevante y actual para garantizar un tratamiento más seguro y efectivo. Se realizó una revisión sistemática de la literatura disponible en las bases de datos: PubMed, Scielo y Science Direct; utilizando los siguientes descriptores: asma, corticosteroides, farmacoterapia y niños. Se incluyeron artículos en la categoría original disponibles en su totalidad, publicados en portugués, inglés y español durante el año 2016 y 2021. De los 233 estudios encontrados, se seleccionaron 14. En la investigación se observó la influencia que tiene el tamaño de partícula sobre la efectividad y seguridad del tratamiento, cambiando la disponibilidad del fármaco en el órgano seleccionado y la dosis administrada para promover el efecto deseado. Además, se observó las particularidades de los CI disponibles en el mercado que pueden utilizarse solos o en combinación con agonistas $\beta 2$ adrenérgicos, en los que permiten una reducción de la dosis de CI utilizada, reduciendo así la incidencia de posibles reacciones adversas a medicamentos (RAM). Es importante destacar que la fluticasona resultó ser uno de los medicamentos más seguros cuando se analizó su influencia en el crecimiento de niños y adolescentes. Finalmente, a pesar de la variedad de estudios en el área, es necesaria una mayor investigación sobre este tema, especialmente aquellos que involucran la influencia del tamaño de partícula en la efectividad y seguridad del tratamiento, así como las principales RAM sobre el tema propuesto.

Palabras clave: Asma; Corticoesteroides; Farmacoterapia.

\section{Introdução}

De acordo com Global Initiative for Asthma (2019), a asma pode ser definida como uma doença com características heterogêneas, ou seja, pode apresentar diversas causalidades e sintomas, sendo, na maioria das vezes, caracterizada por uma inflamação crônica das vias aéreas. Sua sintomatologia pode ser observada pela presença de sibilância, dispneia, opressão torácica e tosse, variando em sua frequência e intensidade.

Essa enfermidade constitui um dos grandes problemas de saúde pública, sendo considerada a doença crônica não transmissível mais comum na infância e adolescência. Estima-se que existam aproximadamente 300 milhões de indivíduos asmáticos em todo o mundo, sendo responsável por cerca de 250 mil mortes por ano (The Global Asthma Report, 2014). Em estudo desenvolvido no Brasil envolvendo 74.589 adolescentes entre 12 e 17 anos de idade, cerca de 9.771 (13,1\%) tinham o diagnóstico de asma ativa, assim considerada uma prevalência alta entre os adolescentes (Kuschnir et al., 2016).

Vários fatores de risco estão associados ao desenvolvimento da asma. Em estudo envolvendo 104 adolescentes entre 13 e 14 anos de idade, foi possível constatar uma maior prevalência da doença entre os indivíduos do sexo masculino, sendo a sua maioria expostos a possíveis fatores de risco, como a presença de carpete em suas casas, além do fumo passivo, animais de estimação e a poluição atmosférica perto do domicílio (De Assis et al., 2019). Em outro estudo, foi avaliado que o peso de nascimento $<2,5$ quilogramas, a utilização de creches ou berçários, o histórico de asma materna e o consumo de mais de 20 cigarros por dia por residentes no mesmo domicílio podem ser fatores de risco associados a presença de sibilância em adolescentes (Fernandes et al., 2018).

Atualmente o tratamento da asma é realizado por meio do uso de corticosteroides inalatórios (CI) associados ou não ao uso de outras classes de fármacos, com intuito de manter o controle da doença e prevenir riscos futuros. De acordo com a Sociedade Brasileira de Pneumologia e Tisiologia (SBPT) (2020), a escolha do melhor agente deve ser pautada nas características inerentes ao paciente, como fatores de risco, preferência por dispositivo, além da efetividade e segurança da conduta terapêutica.

Atualmente, diversas alternativas estão disponíveis para compor o arsenal terapêutico para o tratamento da asma, incluindo, beclometasona, budesonida, ciclesonida, fluticasona e mometasona. Porém, é importante ressaltar que o uso desses medicamentos pode levar ao desenvolvimento de efeitos adversos locais como irritação da garganta, candidíase oral e em 
alguns casos sistêmicas, como redução da densidade mineral óssea, infecções respiratórias e catarata (Global Initiative for Asthma, 2019)

Nesse contexto, devido a elevada prevalência da asma entre crianças e adolescentes, bem como o elevado impacto causado na saúde pública e além dos erros de administração dos CI, esse estudo tem como objetivo investigar na literatura científica o uso de corticosteroides inalatórios no tratamento da asma, com a finalidade de fornecer informações relevantes e atuais para assegurar um tratamento mais seguro e efetivo.

\section{Metodologia}

A revisão integrativa é uma metodologia de pesquisa em saúde a qual possibilita a identificação das melhores e mais atuais evidências científicas, possibilitando ao leitor a avaliação do assunto abordado, referente aos pontos positivos e negativos, com a finalidade de fundamentar propostas de mudanças nas diversas áreas do quesito saúde (De-la-Torre-UgarteGuanilo et al., 2011).

Com isso, esse estudo tem caráter qualitativo, sendo produzida de acordo com as instruções presentes no Preferred Reporting Items for Systematic reviews and Meta-Analyses (PRISMA), o qual faz uso de uma lista de verificação aplicada no processo de revisão, a fim de aprimorar os relatos obtidos (Liberati et al., 2009).

As fases de elaboração do estudo, pesquisa, seleção, extração e processamento dos dados foram realizadas aos pares, ao final, houve discussão entre os revisores com o intuito de selecionar os estudos adequados para a ampliação da pesquisa. Quando necessário, um terceiro revisor foi consultado para deliberação e resolução.

Nesta investigação, as bases eletrônicas de dados sistematicamente explanadas entre março e maio de 2021 foram Scientific Electronic Library Online (Scielo), National Library of Medicine (PubMed) e ScienceDirect. As buscas eletrônicas de dados foram realizadas por meio da combinação dos descritores e palavras-chave, asthma, corticosteroids, drug therapy e children, no idioma inglês, adicionado o operador booleano AND.

Os artigos selecionados foram avaliados com a finalidade de confirmar se descreviam a terapia farmacológica focada para o uso de $\mathrm{CI}$ em crianças e adolescentes asmáticos.

Foram incluídos os artigos na categoria original, disponibilizados na íntegra, publicados nos idiomas português, inglês e espanhol, com intervalo entre os anos de 2016 e 2021. Os dados coletados dos documentos incluíram características farmacodinâmicas, farmacocinéticas, dosagem, interações medicamentosas e efeitos adversos dos CI, além de demonstrar suas aplicações clínicas potenciais no manejo da asma.

Estudos que não descreviam de forma suficiente os dados, ou seja, não apresentam as variáveis desejáveis para o estudo, além daqueles cuja relação com o tema desta revisão bibliográfica não era significativa, foram excluídos. Somado a isso, também foram suprimidos notícias, editoriais, comentários e cartas de apresentação, bem como as duplicatas.

\section{Resultados e Discussão}

Nos bancos de dados mencionados os descritores foram inseridos. Além disso, filtros como o período desejado e tipo de estudo foram acrescidos nas buscas. Dessa forma, foi possível encontrar 233 artigos dos quais, após a seleção e exclusão dos estudos duplicados, 231 resumos foram analisados tendo em vista os critérios de inclusão e exclusão mencionados nessa metodologia. Após essa etapa, apenas 41 estudos foram selecionados para a leitura na integra. Por fim, após leitura cuidadosa e criteriosa, 14 artigos foram introduzidos nesta revisão (Figura 1). 
Figura 1: Fluxograma demostrando o processo de seleção de artigos nas bases de dados utilizadas.
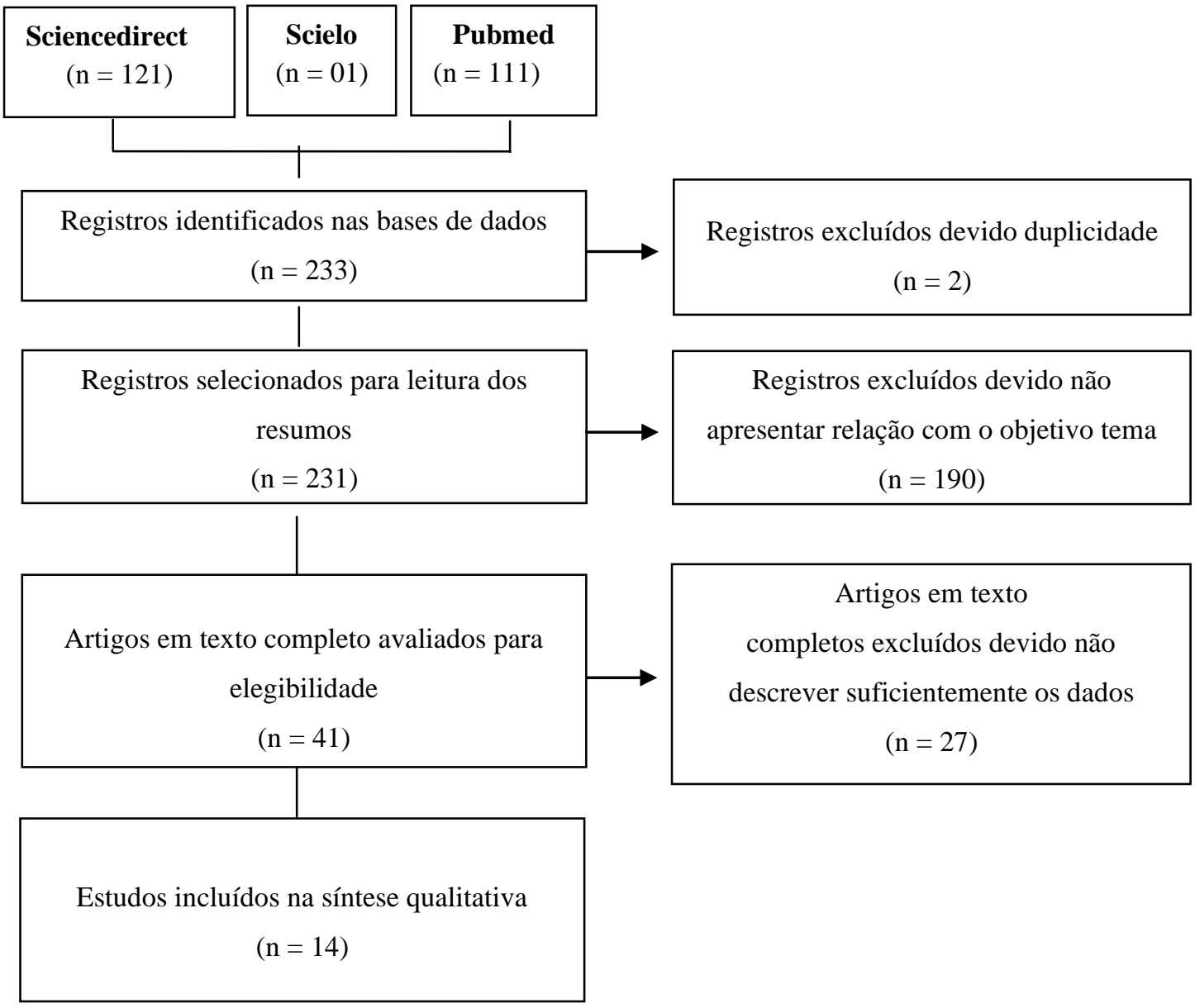

Fonte: Autores.

Com isso, é possível observar no Quadro 1 a composição final dessa revisão, com a titulação dos trabalhos, além dos autores, ano e país, os objetivos e resultados dos estudos selecionados.

Quadro 1: Distribuição dos artigos selecionados.

\begin{tabular}{|c|c|c|}
\hline Título, autor e país & Objetivos do estudo & Resultados \\
\hline $\begin{array}{l}\text { Extrafine Versus Fine Inhaled Corticosteroids in } \\
\text { Relation to Asthma Control: A Systematic } \\
\text { Review and Meta-Analysis of Observational } \\
\text { Real-Life Studies. } \\
\text { Sonnappa, S. et al (2018) } \\
\text { Estados Unidos }\end{array}$ & $\begin{array}{l}\text { Comparar a eficácia de } \\
\text { corticosteroides inalatórios extrafinos } \\
(<2 \mu \mathrm{m}) \text { versus partículas finas }(2-5 \\
\mu \mathrm{m}) \text { para controle da asma }\end{array}$ & $\begin{array}{l}\text { As chances gerais de alcançar o controle da } \\
\text { asma foram significativamente maiores para } \\
\text { corticosteroides inalatórios extrafinos em } \\
\text { comparação com o de partículas finas }\end{array}$ \\
\hline $\begin{array}{l}\text { Effect of inhaled corticosteroid particle size } \\
\text { on asthma efficacy and safety outcomes: a } \\
\text { systematic literature review and meta-analysis } \\
\text { El baou, C. et al. (2017) } \\
\text { Estados Unidos }\end{array}$ & $\begin{array}{l}\text { Comparar o efeito de corticosteroides } \\
\text { inalatórios de tamanho de partículas } \\
\text { extrafinas }(<2 \mu \mathrm{m}) \text { e finas }(2-5 \mu \mathrm{m}) \text { e } \\
\text { sua influência na função pulmonar, } \\
\text { sintomas e segurança em pacientes } \\
\text { com asma }\end{array}$ & $\begin{array}{l}\text { Não foi possível observar diferenças } \\
\text { significativas relevantes em relação ao tamanho } \\
\text { da partícula e os parâmetros avaliados }\end{array}$ \\
\hline $\begin{array}{l}\text { Randomized Trial of Once-Daily Fluticasone } \\
\text { Furoate in Children with Inadequately } \\
\text { Controlled Asthma } \\
\text { Oliver, A. J. et al. (2016) } \\
\text { Estados Unidos }\end{array}$ & $\begin{array}{l}\text { Avaliar a dose-resposta, eficácia e } \\
\text { segurança da fluticasona ( } 25 \mu \mathrm{g}, 50 \\
\mu \mathrm{g} \text { e } 100 \mu \mathrm{g}) \text {, uma vez ao dia, durante } \\
\text { o período de } 12 \text { semanas em crianças } \\
\text { asmáticas }\end{array}$ & $\begin{array}{l}\text { O uso da fluticasona nas três doses avaliadas } \\
\text { mostrou resultados significativos no estudo. Foi } \\
\text { possível observar que o evento adverso mais } \\
\text { presente foi de tosse persistente }\end{array}$ \\
\hline
\end{tabular}




\begin{tabular}{|c|c|c|}
\hline $\begin{array}{l}\text { Comparison of clinical effects of } \\
\text { beclomethasone dipropionate \& budesonide in } \\
\text { treatment of children with mild } \\
\text { persistent asthma: A double-blind, randomized, } \\
\text { controlled study } \\
\text { Singh, A et al. (2016) } \\
\text { India }\end{array}$ & 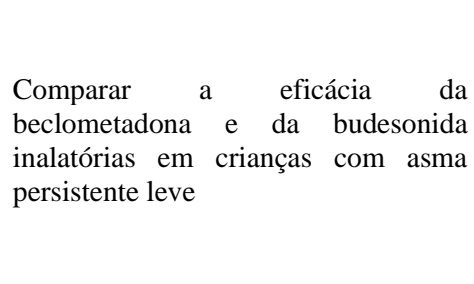 & $\begin{array}{l}\text { Houve uma melhora significativa nos dois } \\
\text { grupos analisados e nenhum evento adverso foi } \\
\text { observado em nenhum dos grupos avaliados }\end{array}$ \\
\hline $\begin{array}{l}\text { Increased versus stable doses } \\
\text { inhaled corticosteroids for exacerbations } \\
\text { chronic asthma in adults and children } \\
\text { Kew, K. M. et al. (2016) } \\
\text { Inglaterra }\end{array}$ & $\begin{array}{l}\text { Comparar a eficácia clínica e } \\
\text { segurança de doses aumentadas e } \\
\text { estáveis de corticosteroides } \\
\text { inalatórios para tratamento domiciliar } \\
\text { de exacerbações em crianças e adultos } \\
\text { com asma persistente }\end{array}$ & $\begin{array}{l}\text { As evidências não mostraram um benefício } \\
\text { significativo ao aumentar a dose de } \\
\text { corticosteroides inalatórios. Além disso, com o } \\
\text { aumento da dose não foi possível observar uma } \\
\text { maior incidência de eventos adversos }\end{array}$ \\
\hline $\begin{array}{l}\text { Efficacy of budesonide/formoterol maintenance } \\
\text { and reliever therapy compared with higher-dose } \\
\text { budesonide as step-up from low-dose } \\
\text { inhaled corticosteroid treatment } \\
\text { Jenkins, C. R. et al (2017) } \\
\text { Austrália }\end{array}$ & $\begin{array}{l}\text { Avaliar a eficácia da combinação de } \\
\text { budesonida-formoterol e melhorar a } \\
\text { taxa de exacerbação, função pulmonar } \\
\text { e uso de alívio em comparação com o } \\
\text { tratamento de referência de uma dose } \\
\text { fixa mais elevada de budesonida }\end{array}$ & $\begin{array}{l}\text { A taxa de exacerbação grave e função pulmonar } \\
\text { foi semelhante para as duas linhas terapêuticas, } \\
\text { entretanto o tratamento com budesonida- } \\
\text { formoterol reduziu a quantidade de } \\
\text { corticosteroide utilizada }\end{array}$ \\
\hline $\begin{array}{l}\text { Inhaled Combined Budesonide-Formoterol as } \\
\text { Needed in Mild Asthma } \\
\text { O'Byrne, P. M. et al (2018) } \\
\text { Estados Unidos }\end{array}$ & $\begin{array}{l}\text { Avaliar a eficácia da combinação de } \\
\text { budesonida-formoterol em relação à } \\
\text { terbutalina e budesonida isoladamente } \\
\text { para a asma bem controlada }\end{array}$ & $\begin{array}{l}\text { Em relação ao tempo de controle da doença, a } \\
\text { budesonida-formoterol foi superior a terbutalina, } \\
\text { mas inferior à terapia com a budesonida isolada. } \\
\text { Além disso, a taxa anual de exacerbação foi } \\
\text { maior para a terbulina, } 0,20 \text {, enquanto para a } \\
\text { budesonida-formoterol e budesonida isolada, } \\
0,07 \text { e } 0,09 \text {, respectivamente }\end{array}$ \\
\hline $\begin{array}{l}\text { Efficacy and safety of budesonide/formoterol } \\
\text { pMDI vs budesonide pMDI in } \\
\text { asthmatic children }(6-<12 \text { years }) \\
\text { Pearlman, D. S. et al }(2017) \\
\text { Estados Unidos }\end{array}$ & $\begin{array}{l}\text { Avaliar a eficácia de budesonida com } \\
\text { combinaçóes fixas versus budesonida } \\
\text { isolada em crianças com asma de } 6 \text { a } \\
12 \text { anos de idade }\end{array}$ & $\begin{array}{l}\text { A combinação de budesonida-formoterol } \\
\text { mostrou melhorias na função pulmonar } \\
\text { estatisticamente e clinicamente significativas } \\
\text { versus a budesonida isolada }\end{array}$ \\
\hline 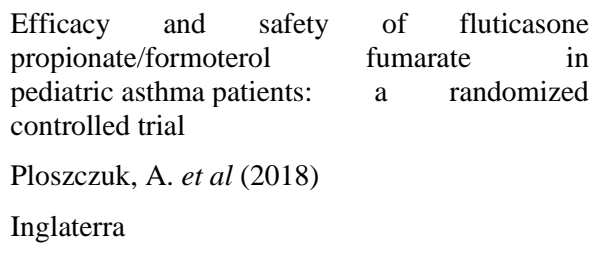 & $\begin{array}{l}\text { Demonstrar superioridade de eficácia } \\
\text { da combinação de fluticasona- } \\
\text { formoterol em relação à fluticasona } \\
\text { isolada e a não inferioridade em } \\
\text { relação à fluticasona-salmeterol }\end{array}$ & $\begin{array}{l}\text { Observou-se que a fluticasona-formoterol } \\
\text { apresentou eficácia superior à fluticasona } \\
\text { isolada em termos de função pulmonar, além } \\
\text { disso, a associação de fluticasona-formoterol } \\
\text { não foi inferior a fluticasona-salmeterol }\end{array}$ \\
\hline $\begin{array}{l}\text { Corticosteroid plus beta (2)-agonist in a single } \\
\text { inhaler as reliever therapy in intermittent and } \\
\text { mild asthma: a proof-of-concept systematic } \\
\text { review and meta-analysis } \\
\text { Wang, G. et al (2017) } \\
\text { China }\end{array}$ & $\begin{array}{l}\text { Explorar sistematicamente a eficácia e } \\
\text { segurança de corticosteroide } \\
\text { inalatório associado a } \beta_{2} \text {-agonista } \\
\text { adrenérgico de ação rápida para o } \\
\text { alívio de asma intermitente e } \\
\text { persistente leve }\end{array}$ & $\begin{array}{l}\text { A terapia de corticosteroide inalatório associado } \\
\text { a beta-agonista adrenérgico é mais efetiva que } \\
\text { apenas com o uso do } \beta_{2} \text {-agonista adrenérgico. } \\
\text { Entretanto, indiferente quando comparado com } \\
\text { os corticosteroides inalatórios isolados, além } \\
\text { disso, essa terapia aumenta a exposição de } \\
\text { corticoides de } 2 \text { a } 3 \text { vezes quando comparado ao } \\
\text { tratamento com corticosteroide inalatório mais } \\
\beta_{2} \text {-agonista adrenérgico }\end{array}$ \\
\hline $\begin{array}{l}\text { A systematic review of adverse drug events } \\
\text { associated with administration of } \\
\text { common asthma medications in children } \\
\text { Leung, J. S. et al. (2017) } \\
\text { Canadá }\end{array}$ & $\begin{array}{l}\text { Determinar as frequências de eventos } \\
\text { adversos em medicamentos } \\
\text { pediátricos para asma }\end{array}$ & $\begin{array}{l}\text { Várias classes de medicamentos foram } \\
\text { encontradas no estudo, entre eles, } \\
\text { corticosteroides inalatórios. Foi possível } \\
\text { observar a presença de } 174 \text { eventos adversos, } \\
\text { afetando } 13 \text { sistemas de órgãos, incluindo a } \\
\text { supra-renal e supressão do crescimento }\end{array}$ \\
\hline $\begin{array}{l}\text { Knemometry Assessment of Short-term Growth } \\
\text { in Children With Asthma Receiving Fluticasone } \\
\text { Furoate for } 2 \text { Weeks: A Randomized, Placebo- } \\
\text { controlled, Crossover Trial } \\
\text { Wolthers, O. D. et al (2017) } \\
\text { Dinamarca }\end{array}$ & $\begin{array}{l}\text { Avaliar o crescimento de curto prazo } \\
\text { da perna em crianças fazendo o uso } \\
\text { de fluticasona inalado }(50 \mu \mathrm{g}) \text {, por } \\
\text { duas semanas, versus placebo }\end{array}$ & $\begin{array}{l}\text { A taxa de crescimento médio em relação a } \\
\text { fluticasona foi de } 0,31 \mathrm{~mm} / \mathrm{semana} \text {, no caso do } \\
\text { placebo, foi de } 0,36 \mathrm{~mm} / \mathrm{semana}\end{array}$ \\
\hline
\end{tabular}




\begin{tabular}{|c|c|c|}
\hline $\begin{array}{l}\text { Inhaled corticosteroids in children with } \\
\text { persistent asthma: effects of different drugs and } \\
\text { delivery devices on growth } \\
\text { Axelsson, I. et al (2019) } \\
\text { Suécia }\end{array}$ & $\begin{array}{l}\text { Avaliar o impacto de diferentes } \\
\text { corticosteroides inalatórios e } \\
\text { dispositivos de aplicação no } \\
\text { crescimento linear de crianças com } \\
\text { asma persistente }\end{array}$ & $\begin{array}{l}\text { Em doses equivalentes, a fluticasona possui uma } \\
\text { velocidade de crescimento linear } \\
\text { significativamente maior que a beclometasona. } \\
\text { Além disso, a fluticasona dada em uma dose } \\
\text { equivalente teve um efeito menos supressor do } \\
\text { que a budesonida no crescimento infantil }\end{array}$ \\
\hline $\begin{array}{l}\text { Serious Asthma Events with Budesonide plus } \\
\text { Formoterol vs. Budesonide Alone } \\
\text { Peters S. P. et al (2016) } \\
\text { Estados Unidos }\end{array}$ & $\begin{array}{l}\text { Avaliar os riscos de reações adversas } \\
\text { em decorrência da adição de } \\
\text { formoterol a terapia de manutenção } \\
\text { com budesonida para tratamento da } \\
\text { asma }\end{array}$ & $\begin{array}{l}\text { O estudo demonstrou que dos } 5.846 \text { indivíduos } \\
\text { que estavam associando a budesonida com o } \\
\text { formoterol, } 43 \text { apresentaram evento adverso } \\
\text { grave. Enquanto, que os } 5.847 \text { pacientes que } \\
\text { utilizavam a budesonida isolada, } 40 \\
\text { apresentaram evento adverso grave }\end{array}$ \\
\hline
\end{tabular}

Fonte: Autores.

A asma não controlada em crianças é potencialmente preocupante, pois pode afetar diretamente a qualidade de vida desses pacientes, retardando seu crescimento, influenciando diretamente no sono, além da prática de exercícios físicos e ausência escolar (Oliver et al., 2016). Assim, é bem descrito nas diretrizes nacionais e internacionais a importância do uso de CI para o tratamento dessa enfermidade em crianças (Singh et al., 2016).

A partir dos dados obtidos nessa pesquisa é possível categorizar essa discussão nos seguintes tópicos: influência do tamanho de partícula na efetividade do fármaco, corticosteroides inalatórios isolados, associação de corticosteroides e $\beta_{2}$ agonistas adrenérgicos, e principais eventos adversos relacionados ao uso de CI.

\section{Influência do tamanho de partícula na efetividade do fármaco}

O tamanho de partícula do fármaco pode ser considerado como um dos fatores que mais influenciam a administração dos medicamentos inalatórios, pois pode interferir na disponibilidade das drogas ao seu local de ação e assim modificar o efeito desejado, interferindo diretamente na efetividade e segurança do tratamento proposto para a asma (Sonnappa et al.,2018; El baou et al., 2017).

Um estudo se propôs a comparar a influência do tamanho de partículas extrafina $(<2 \mu \mathrm{m})$ e fina $(2-5 \mu \mathrm{m})$ de CI para o tratamento da asma, nesse não foi possível constatar nenhuma diferença significativa quando avaliados os parâmetros pulmonares propostos pelo estudo, pois apesar de se obter uma maior deposição do medicamento na porção distal do pulmão, isso não influenciou em melhores resultados clínicos para os pacientes asmáticos. Porém, é importante ressaltar que quanto menor for o tamanho de partícula menores vão ser as chances de deposição orofaríngea do fármaco, diminuindo assim o risco de reações adversas localizadas nessa região (El baou et al., 2017).

Entretanto, uma meta-análise de estudos observacionais foi realizada com o objetivo de comparar a eficácia de CI de partículas extrafinas e finas para estimar influência do tamanho de partícula no tratamento de pessoas asmáticas. Durante o processo da pesquisa, foi possível observar nas bases de dados analisadas a presença de 6 estudos envolvendo o propionato de beclometasona extrafino $(<2 \mu \mathrm{m})$ e 1 estudo envolvendo esse mesmo fármaco, além da ciclesonida extrafina $(<2 \mu \mathrm{m})$, comparando todos esses fármacos com amostras de partículas finas (2-5 $\mu \mathrm{m})$ (Sonnappa et al.,2018).

Foi possível constatar que o tamanho de partícula pode influenciar diretamente na deposição do fármaco em seu local de ação, aumentando a efetividade do tratamento e diminuindo a quantidade de fármaco necessária para a ação desejada. Assim as partículas extrafinas possuem chances significativamente maiores de alcançar o controle da asma, reduzindo a frequência de exacerbação da doença e doses utilizadas no tratamento proposto, diminuindo assim as chances de reações adversas sistêmicas (Sonnappa et al.,2018). 


\section{Corticosteroides inalatórios isolados}

Um dos CI mais utilizados para a asma na prática clínica é a fluticasona. Um estudo avaliou a dose-resposta, a eficácia e a segurança de 3 doses desse medicamento $(25 \mu \mathrm{g}, 50 \mu \mathrm{g}$ e $100 \mu \mathrm{g})$, sendo administrado uma vez ao dia por um período de tratamento de 12 semanas, para crianças com idade entre 5-11 anos com asma não controlada. Foi possível constatar que todos os tratamentos propostos pela pesquisa resultaram em efeitos positivos na função pulmonar, independente da dose. Entretanto, não foi possível perceber o efeito aparente com a variação da dose do fármaco, possivelmente resultante do método de análise da efetividade do fármaco. Assim, esse fármaco pode ser uma opção terapêutica para crianças que são acometidas com asma não controlada (Oliver et al., 2016).

A budesonida e a beclometasona são medicamentos bastante utilizados devido sua relação custo-eficácia, entretanto sempre surgem questionamentos referentes a qual desses medicamentos apresenta melhores características. Tendo em vista essa problemática, um estudo comparou a eficácia da budesonida $400 \mu \mathrm{g} / \mathrm{dia}$ versus beclometasona $400 \mu \mathrm{g} / \mathrm{dia}$ em crianças. Foi possível observar que a budesonida apresentou melhores resultados pulmonares que a beclometasona, pois relatou-se que os pacientes que fizeram o uso da budesonida apresentaram uma maior melhora no volume expiratório forçado, entretanto, quando comparado em relação a frequência de sintomas após o tratamento, as amostras analisadas se mostraram equivalentes (Singh et al., 2016).

Após a avaliação desses dados, foi possível constatar que houve uma melhora na qualidade de vidas dessas crianças, principalmente aquelas que utilizaram a budesonida como linha de tratamento, pois houve redução na interrupção do sono e menos idas à urgência por crises asmáticas. Assim, apesar da budesonida ser ligeiramente mais eficaz que a beclometasona, o estudo avaliado sugere que as duas linhas de tratamento são eficazes para o tratamento de crianças acometidas por asma (Singh et al., 2016).

Um meta-análise se propôs comparar a eficácia clínica de doses aumentadas e estáveis de CI, com a finalidade de avaliar a incidência de exacerbações da asma após o tratamento com essas doses variáveis de medicamentos. Nesse estudo, percebeu-se que a dose não foi o fator determinante para a diminuição de incidência de exacerbações, pois a efetividade do tratamento não melhorou com o aumento das doses dos fármacos. Assim, as evidências cientificas atuais não apoiam o aumento da dose dos CI para o tratamento de asma em crianças (Kew et al., 2016).

\section{Associação de corticosteroides e $\beta 2$-agonista adrenérgico}

As diretrizes clínicas referentes ao manejo da asma sugerem que os CI isolados em baixa dosagem sejam a primeira linha de escolha para o tratamento de manutenção dessa doença. Entretanto, quando o tratamento não está sendo efetivo, é necessário que seja acrescido um novo medicamento, sendo indicado, na maioria das vezes, um $\beta_{2}$-agonista adrenérgico. É importante ressaltar que os $\beta_{2}$-agonista adrenérgicos não apresentam atividade anti-inflamatória, justificando a importância de que sejam utilizados com os CI em associação partindo do princípio de que a asma é uma doença caracterizada pela inflamação das vias aéreas (O’Byrne et al., 2018; Wang et al., 2017; Jenkins et al., 2017; Peters et al., 2016).

Ao comparar a eficácia e a segurança da budesonida $(200 \mu \mathrm{g})$ duas vezes ao dia, terbutalina $(0,5 \mathrm{mg})$ e a associação de budesonida-formoterol (200 $\mu \mathrm{g}-6 \mu \mathrm{g})$, esses dois últimos utilizados quando necessário. Constatou-se que a efetividade da combinação de budesonida-formoterol é superior a utilização do $\beta_{2}$-agonista adrenérgico isolado. Além disso, foi possível observar que o tratamento com a budesonida-formoterol é tão eficaz quanto a budesonida isolada, a não ser quando avaliado a quantidade de semanas com asma bem controlada, onde o CI isolado apresentou uma superioridade de semanas com a doença bem controlada (O’Byrne et al., 2018).

É importante ressaltar que o tratamento com a combinação dos medicamentos reduziu drasticamente a quantidade de CI utilizado. Portanto, a utilização da combinação de budesonida-formoterol pode ser uma alternativa promissora para o 
tratamento da asma, pois reduz substancialmente a quantidade de CI utilizado, diminuindo assim a chance de reações adversas decorrentes de CI, sendo essa uma das principais causas de abandono do tratamento (O'Byrne et al., 2018).

Em estudo semelhante, foi possível reafirmar a superioridade da eficácia da combinação de budesonida-formoterol $(160 \mu \mathrm{g}-9 \mu \mathrm{g})$ em detrimento da budesonida isolada (160 $\mu \mathrm{g})$. A incidência de exarcebações e melhorias nos resultados no que se refere aos sintomas e qualidade de vida das crianças foi semelhante, entretanto a associação do CI com o $\beta_{2}$-agonista adrenérgico resultou em melhores resultados clinicamente significantes na função pulmonar de crianças entre 6 e 12 anos de idade (Pearlman et al., 2017).

O formoterol é o $\beta_{2}$-agonista adrenérgico de ação mais rápida, tendo velocidade de início de ação comparada ao salbutamol, um $\beta_{2}$-agonista adrenérgico de ação curta. Esses fármacos são utilizados, na grande maioria das vezes, associados a fluticasona, um CI eficaz e com efeitos anti-inflamatórios sustentados. Quando comparados o uso da combinação de fluticasona-formoterol (100 $\mu \mathrm{g}-10 \mu \mathrm{g})$, fluticasona-salbutamol $(100 \mu \mathrm{g}-50 \mu \mathrm{g})$ e a fluticasona $(100 \mu \mathrm{g})$ isolada em uma população pediátrica é possível perceber, levando em consideração parâmetros envolvendo a função pulmonar, que o tratamento realizado com a fluticasona-formoterol foi mais efetivo frente a combinação de fluticasona-salbutamol e a fluticasona isolada. Assim, esse estudo confirma a superioridade na efetividade da combinação de fluticasona-formoterol, em detrimento, principalmente, da fluticasona isolada (Ploszczuk et al., 2018).

$\mathrm{O}$ uso de $\beta_{2}$-agonista de curta duração é imprescindível para aliviar rapidamente os sintomas da asma, entretanto não apresentam atividade anti-inflamatória significativa. Sabendo disso, um estudo se propôs a avaliar sistematicamente a eficácia e segurança do uso de CI associados $\beta_{2}$-agonista de curta duração, como monoterapia de alivio em crianças e adultos com asma persistente. Assim, a utilização desse esquema terapêutico se mostrou como uma opção efetiva e segura, partindo do principio que a combinação reduziu significativamente o risco de exacerbação, despertar noturno e influência a longo prazo sobre o crescimento de crianças e adolescentes, já que esse esquema terapêutico diminui de 2 a 5 vezes a concentração de CI utilizado, fator bem descrito na literatura, referente a influência de corticosteroides sobre o crescimento linear. Por último, como o esquema mencionado esta presente em um único dispositivo inalatório a comodidade posológica é melhorada, que por consequência influencia diretamente na adesão terapêutica (Wang et al., 2017).

\section{Principais eventos adversos relacionado ao uso de CI}

A prevalência de reações adversas com uso de CI está intimamente ligada a quantidade de fármaco que chega à corrente sanguínea de forma inalterada, ou seja, com a biodisponibilidade sistêmica desse medicamento. Dessa forma, vale ressaltar que quanto menor for a dose de CI, menores serão as chances de ocorrência de reações adversas à medicamentos (RAM) (Axelsson et al., 2019).

Os CI são considerados medicamentos relativamente seguros para asma, entretanto, a utilização desses a longo prazo podem ser motivos de preocupação, partindo do princípio que esses fármacos podem influenciar na segurança do tratamento e aumentar a incidência de RAM. Entre as reações adversas mais descritas na literatura para essa classe de medicamentos, a supressão do crescimento é um risco potencialmente importante na população pediátrica, além disso, os CI podem causar alterações na adrenal e até efeitos neurotóxicos (Axelsson et al., 2019; Wolthers et al., 2017).

Treze sistemas de órgãos do corpo humano podem ser afetados pelos CI. Entre eles a supressão da adrenal é bem descrita com a fluticasona, budesonida e ciclesonida, alterando principalmente a concentração do hormônio adrenocorticotrófico e do cortisol. Além disso, comprometimentos dose-dependentes podem influenciar no crescimento linear de crianças e adolescentes, influenciando na altura final do adulto (Leung et al., 2017).

Foi realizado um estudo com o objetivo de avaliar o efeito do furoato de fluticasona (50 $\mu \mathrm{g})$ sobre a influência no crescimento em crianças entre 5-11 anos, durante um curto período. Nesse estudo, foi possível constatar que o crescimento de 
crianças fazendo o uso do CI não apresentou alterações estatisticamente significativas. Entretanto, isso pode ter acontecido devido ao curto período de tempo analisado, ou seja, apenas 2 semanas. Contudo, acredita-se que o furoato de fluticasona possui uma alta afinidade para os receptores de glicocorticoides, assim doses menores são capazes de gerar o efeito desejável e, apresenta ainda, como particularidade, algumas alterações físico-químicas que permitem que esse fármaco tenha uma maior retenção pulmonar e baixa biodisponibilidade sistêmica, diminuindo assim a exposição sistêmica e reduzindo a interferência sobre o crescimento na população pediátrica (Wolthers et al., 2017).

Uma meta-análise foi realizada com o objetivo de avaliar o impacto do crescimento em diferentes CI em crianças asmáticas, entre 4-12 anos. Constatou-se que quando comparada a budesonida com a fluticasona, administrado em doses equivalentes, durante um período entre 12 semanas a 1 ano, a fluticasona apresentou menos efeito de supressão no crescimento que a budesonida. Além disso, quando comparada, a velocidade de crescimento linear entre a fluticasona e a beclometasona, a fluticasona apresentou resultados mais satisfatórios, reafirmando mais uma vez sua menor interferência no crescimento de crianças e adolescentes (Axelsson et al., 2019).

Ainda existem muitas preocupações referentes a segurança da associação de CI e $\beta_{2}$-agonista adrenérgico. Sabendo disso, um estudo com o objetivo de avaliar se a adição de formoterol a terapia de manutenção com budesonida poderia aumentar a incidência de RAM graves. Entre os 5.846 pacientes que receberam budesonida-formoterol, 43 apresentaram RAM graves versus 40 indivíduos dos 5.847 que estavam fazendo uso da budesonida isolada. Assim, a adição de formoterol a monoterapia de budesonida não pareceu aumentar os riscos de RAM, sendo, portanto, uma alternativa segura de associação (Peters et al., 2016).

As principais limitações encontradas no decorrer da realização do estudo foram os poucos estudos encontrados na literatura cientifica, principalmente acerca da influência do tamanho de partícula sobre a farmacoterapia dos CI e as principais reações adversas encontradas na população pediátrica. Em relação aos pontos fortes, esse estudo pode ajudar a direcionar uma farmacoterapia mais efetiva e principalmente mais segura para as crianças e adolescentes, partindo do princípio da importância que a associação entre fármacos apresenta, reduzindo a dose dos CI e diminuindo a incidência de reações adversas a medicamentos (RAM).

\section{Conclusão}

$\mathrm{Na}$ pesquisa observou-se a influência que o tamanho de partícula tem sobre a efetividade e segurança do tratamento, alterando a disponibilidade do medicamento no órgão alvo e até a dosagem administrada para o efeito desejado. Ademais, as particularidades dos CI disponíveis no mercado que podem ser utilizados isoladamente ou em combinações com $\beta_{2}$-agonistas adrenérgicos, nas quais essas permitem uma redução da dose de CI usado, diminuindo assim a incidência de possíveis RAM. É interessante salientar que, comparada aos demais medicamentos, a fluticasona apresentou-se como um dos mais seguros no que tange sua influência sobre o crescimento de crianças e adolescentes. Por fim, apesar da quantidade de estudos na área, a realização de novas pesquisas acerca do tema é necessária, essencialmente aqueles que tangem a influência que o tamanho de partícula tem sobre a efetividade e segurança do tratamento, bem como as principais RAM a respeito do tema proposto.

\section{Referências}

Axelsson, I., Naumburg, E., Prietsch, S. O., \& Zhang, L. (2019). Inhaled corticosteroids in children with persistent asthma: effects of different drugs and delivery devices on growth. Cochrane Database of Systematic Reviews, (6).

De Assis, E. V., Santana, M. D., do Feitosa, A., \& de Sousa, M. N. A. (2019). Prevalência de sintomas de asma e fatores de risco em adolescentes. J Hum Growth Dev, 29(1), 110-116.

De-la-Torre-Ugarte, M. C., Takahashi, R. F., \& Bertolozzi, M. R. (2011). Revisão sistemática: noções gerais. Revista da Escola de Enfermagem da USP, 45(5), 1260-1266. 
El Baou, C., Di Santostefano, R. L., Alfonso-Cristancho, R., Suarez, E. A., Stempel, D., Everard, M. L., \& Barnes, N. (2017). Effect of inhaled corticosteroid particle size on asthma efficacy and safety outcomes: a systematic literature review and meta-analysis. BMC pulmonary medicine, 17(1), 1-16.

Fernandes, S. D. S. C., Solé, D., Camargos, P., Andrade, C. R. D., \& Ibiapina, C. D. C. (2018). Fatores associados à expressão da asma em adolescentes. Jornal Brasileiro de Pneumologia, 44(1), 12-17.

Global Initiative for Asthma. Global for Asthma Management and prevention (2019).: https://ginasthma.org/wp-content/uploads/2019/06/GINA-2019-mainreport-June-2019-wms.pdf

Jenkins, C. R., Eriksson, G., Bateman, E. D., Reddel, H. K., Sears, M. R., Lindberg, M., \& O’Byrne, P. M. (2017). Efficacy of budesonide/formoterol maintenance and reliever therapy compared with higher-dose budesonide as step-up from low-dose inhaled corticosteroid treatment. BMC pulmonary medicine, 17(1), 1-8.

Kew, K. M., Quinn, M., Quon, B. S., \& Ducharme, F. M. (2016). Increased versus stable doses of inhaled corticosteroids for exacerbations of chronic asthma in adults and children. Cochrane Database of Systematic Reviews, (6).

Kuschnir, F. C., Gurgel, R. Q., Solé, D., Costa, E., Felix, M. M. R., Oliveira, C. L. D., Vasconcellos, M. T. L., \& Kuschnir, M. C. C. (2016). ERICA: prevalência de asma em adolescentes brasileiros. Revista de Saúde Pública, 50, 13s.

Leung, J. S., Johnson, D. W., Sperou, A. J., Crotts, J., Saude, E., Hartling, L., \& Stang, A. (2017). A systematic review of adverse drug events associated with administration of common asthma medications in children. PLoS One, 12(8), e0182738.

Liberati, A., Altman, D. G., Tetzlaff, J., Mulrow, C., Gotzsche, P. C., Ioannidis, J. P. A., Clarke, M., Devereaux, P. J., Kleijnen, J., \& Moher, D. (2009). The PRISMA statement for reporting systematic reviews and meta-analyses of studies that evaluate healthcare interventions: explanation and elaboration. $B M J$, $339, \mathrm{~b} 2700$.

O’Byrne, P. M., FitzGerald, J. M., Bateman, E. D., Barnes, P. J., Zhong, N., Keen, C., Jorup, C., Lamarca, R., Inavov, S., \& Reddel, H. K. (2018). Inhaled combined budesonide-formoterol as needed in mild asthma. New England Journal of Medicine, 378(20), 1865-1876.

Oliver, A. J., Covar, R. A., Goldfrad, C. H., Klein, R. M., Pedersen, S. E., Sorkness, C. A., Tomkins, S. A., Villarán, C., \& Grigg, J. (2016). Randomized trial of once-daily fluticasone furoate in children with inadequately controlled asthma. The Journal of pediatrics, 178, 246-253.

Pearlman, D. S., Eckerwall, G., McLaren, J., Lamarca, R., Puu, M., Gilbert, I., Jorup, C., Sandin, K., \& Lanz, M. J. (2017). Efficacy and safety of budesonide/formoterol pMDI vs budesonide pMDI in asthmatic children (6-<12 years). Annals of Allergy, Asthma \& Immunology, 118(4), 489-499.

Peters, S. P., Bleecker, E. R., Canonica, G. W., Park, Y. B., Ramirez, R., Hollis, S., Fjallbrant, H., Jorup, C., \& Martin, U. J. (2016). Serious asthma events with budesonide plus formoterol vs. budesonide alone. New England Journal of Medicine, 375(9), 850-860.

Pizzichini, M. M. M., Carvalho-Pinto, R. M. D., Cançado, J. E. D., Rubin, A. S., Cerci Neto, A., Cardoso, A. P., Cruz, A. A., Fernandes, A. L. G., Blanco, D. C., Vianna, E. O., Junior, G. C., Rizzo, J. A., Fritscher, L. G., Caetano, L. S. B., Pereira, L. F. F., Rabahi, M. F., Oliveira, M. A., Lima, M. A., Almeida, M. B., Stelmach, R., Pitrez, P. M., \& Cukier, A. (2020). Recomendações para o manejo da asma da Sociedade Brasileira de Pneumologia e Tisiologia-2020. Jornal Brasileiro de Pneumologia, 46(1).

Płoszczuk, A., Bosheva, M., Spooner, K., McIver, T., \& Dissanayake, S. (2018). Efficacy and safety of fluticasone propionate/formoterol fumarate in pediatric asthma patients: a randomized controlled trial. Therapeutic advances in respiratory disease, 12, 1753466618777924.

Prishtina, L. N. (2014). The Global Asthama Report 2014: https://rsdjournal.org/index.php/rsd/about/submissions

Singh, A., Nandan, D., Dewan, V., \& Sankar, J. (2016). Comparison of clinical effects of beclomethasone dipropionate \& budesonide in treatment of children with mild persistent asthma: A double-blind, randomized, controlled study. The Indian journal of medical research, 144(2), 250.

Sonnappa, S., McQueen, B., Postma, D. S., Martin, R. J., Roche, N., Grigg, J., Guilbert, T., Gouder, C., Pizzichini, E., Niimi, A., Phipatanakul, W., Chisholm, A., Dandurand, R. J., Kaplan, A., Israel, E., Papi, A., Aalderen, W. M. C., Usmani, O. S.,\& Price, D. B. (2018). Extrafine versus fine inhaled corticosteroids in relation to asthma control: a systematic review and meta-analysis of observational real-life studies. The Journal of Allergy and Clinical Immunology: In Practice, 6(3), 907-915.

Wang, G., Zhang, X., Zhang, H. P., Wang, L., Kang, D. Y., \& Barnes, P. J. (2017). Corticosteroid plus $\beta$ 2-agonist in a single inhaler as reliever therapy in intermittent and mild asthma: a proof-of-concept systematic review and meta-analysis. Respiratory research, 18(1), 1-18.

Wolthers, O. D., Stone, S., Bareille, P., Tomkins, S., \& Khindri, S. (2017). Knemometry assessment of short-term growth in children with asthma receiving fluticasone furoate for 2 weeks: a randomized, placebo-controlled, crossover trial. Clinical therapeutics, 39(6), 1191-1199. 